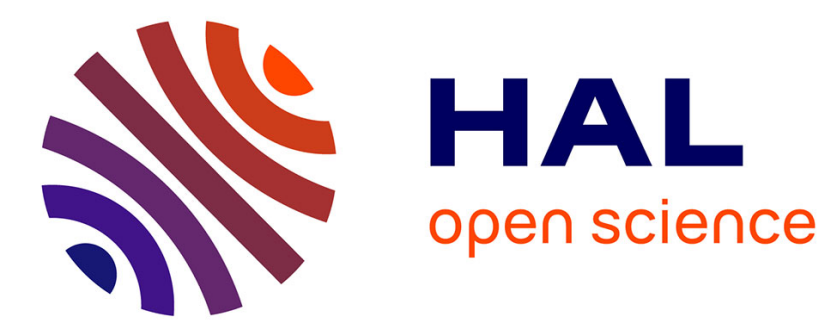

\title{
Protection performance of bicycle helmets
}

Caroline Deck, Nicolas Bourdet, Frank Meyer, Rémy Willinger

\section{To cite this version:}

Caroline Deck, Nicolas Bourdet, Frank Meyer, Rémy Willinger. Protection performance of bicycle helmets. Journal of Safety Research, 2019, 71, pp.67 - 77. 10.1016/j.jsr.2019.09.003 . hal-03488470

\section{HAL Id: hal-03488470 \\ https://hal.science/hal-03488470}

Submitted on 21 Dec 2021

HAL is a multi-disciplinary open access archive for the deposit and dissemination of scientific research documents, whether they are published or not. The documents may come from teaching and research institutions in France or abroad, or from public or private research centers.
L'archive ouverte pluridisciplinaire HAL, est destinée au dépôt et à la diffusion de documents scientifiques de niveau recherche, publiés ou non, émanant des établissements d'enseignement et de recherche français ou étrangers, des laboratoires publics ou privés.

\section{다)(1) $\$$}

Distributed under a Creative Commons Attribution - NonCommercial| 4.0 International 


\author{
Caroline DECK \\ Ph.D. Researcher \\ University of Strasbourg, ICube, UMR 7357, \\ Multiscale Materials and Biomechanics \\ 2 , rue Boussingault \\ 67000 Strasbourg, France \\ Phone: +33 368852940 \\ E-mail: \\ deck@unistra.fr
}

\title{
Protection performance of bicycle helmets
}

Authors' information:

1. Caroline DECK, Ph.D. Researcher,

University of Strasbourg, Icube, UMR 7357 Multiscale Materials and Biomechanics, 2 rue Boussingault, 67000 Strasbourg, France.

E-mail: deck@unistra.fr

2. Nicolas BOURDET, Ph.D. Researcher,

University of Strasbourg, Icube, UMR 7357 Multiscale Materials and Biomechanics, 2 rue Boussingault, 67000 Strasbourg, France.

E-mail: bourdet@unistra.fr

3. Frank MEYER, Ph.D. Researcher,

University of Strasbourg, Icube, UMR 7357 Multiscale Materials and Biomechanics, 2 rue Boussingault, 67000 Strasbourg, France.

E-mail: frmeyer@unistra.fr

4. Rémy WILLINGER, Professor

University of Strasbourg, Icube, UMR 7357 Multiscale Materials and Biomechanics, 2 rue Boussingault, 67000 Strasbourg, France.

E-mail: remy.willinger@unistra.fr

We are willing to mention there are no conflicts of interest for that manuscript. 


\title{
Protection performance of bicycle helmets
}

\begin{abstract}
Introduction: The evaluation of head protection systems needs proper knowledge of the head impact conditions in terms of impact speed and angle, as well as a realistic estimation of brain tolerance limits. In current bicycle helmet test procedures, both of these aspects should be improved. Method: The present paper suggests a bicycle helmet evaluation methodology based on realistic impact conditions and consideration of tissue level brain injury risk, in addition to well known headform kinematic parameters. The method is then applied to a set of 32 existing helmets, leading to a total of 576 experimental impact tests followed by 576 numerical simulations of the brain response. Results: It is shown that the most critical impacts are the linear-lateral ones as well as the oblique impact leading to rotation around the vertical axis (ZRot), leading both to around 50\% risks of moderate neurological injuries. Based on this test method, the study enables us to compare the protection capability of a given helmet and eventually to compare helmets via a dedicated rating system.
\end{abstract}

Keywords: Bicycle helmet, Helmet test method, Model based head injury criteria, oblique impacts 


\section{INTRODUCTION}

Cyclists in Europe make around 50 million trips per day and this figure is increasing, especially in urban environment. Accident statistics show that between 2,000 and 2,400 fatalities occur from bike accidents every year in the EU since 2008 (European Road Safety Observatory, 2018). This study also shows that around $8 \%$ of all European road victims are cyclists. Finally it reports that the head is the most frequent injured body part $(25 \%)$ in bike accidents and the top 10 of injury in cyclists include contusion $(31 \%)$, concussion $(6 \%)$, and other specified brain injury $(2 \%)$.

There are several ways to reduce the number of head injuries, such as reducing the frequency of accidents by active traffic planning and education of road users. Where accidents have occurred, the helmet has been shown to provide significant protection (Amoros et al., 2012; Attewell et al., 2012; Povey et al., 1999; Thomson et al., 2000), an observation confirmed based on experimental data by Fahlstedt et al. (2014) and computer simulations (McNally \& Whitehead, 2013).

According to accident analysis reported by Otte (1989), the majority of bicycle helmet impacts are oblique and involve both linear and angular head accelerations. Further, Bourdet et al (2013) demonstrated the oblique characteristic of the head impact velocity vector via accident simulation of 24 bicyclist accident cases. The results from this study showed that the average velocity of the bicyclist head just before impact was $6.8 \pm 2.7 \mathrm{~m} / \mathrm{s}$ and the impact angle was about $33 \pm 20$ degrees. In another study, Verschueren (2009) presented results from 22 bicycle accident reconstructions and found that the average head velocity was between $6.0-7.7 \mathrm{~m} / \mathrm{s}$ with head impact angles about 40-50 degrees. On the other hand Holbourn (1943) suggests that the head rotational acceleration induced by an impact causes high shear strains in the brain leading to cerebral concussion. This observation is confirmed by Gennarelli et al. (1972) (1982) and Ommaya et al. (2002) based on animal model as well as by Deck et al. (2007) and Weaver et al. (2012) based on numerical modelling. Despite this widely recognized understanding of head rotational loading and its effect on brain injury risk, no standard related to bicycle helmet is currently considering head rotational acceleration.

The mandatory EN1078 standard test (2012) for bicycle helmets sold in Europe only captures linear acceleration resulting from linear impacts. The standard's shock absorption section recommends evaluating helmets through drop tests where bicycle helmets are fitted onto an ISO-960 headform equipped with linear accelerometeres sensors and a pass/fail criterion of $250 \mathrm{~g}$ for the maximum linear acceleration. The objective of this bicycle helmet standard is to prevent life-threatening injuries based on the Wayne State University tolerance curve (Lissner et al., 1960; Gurdjian et al., 1953; 1961). Considering recent knowledge, it is possible to evaluate bicycle helmets under more realistic impact conditions and by integrating brain injury criteria considering complex loadings and reversible injury levels.

With this perspective, a number of attempts concerning bicycle helmet evaluation under tangential impacts conditions have been proposed in the literature. Mills and Gilchrist (2008) developed a test rig that consists of a free-falling headform impacting a horizontally moving plate and measuring the headform linear and angular accelerations. The movable plate has drawbacks, as it is not easy to maintain a constant speed of the plate during the impact. McIntosh et al. (2013) used an impact rig that included a drop assembly with a Hybrid III head and neck. The head struck a horizontally moving striker plate. Again, head linear and angular acceleration are recorded as well as the striker plate force. The main limitation of this method is the use of a Hybrid III neck known to be very stiff compared to the human neck. Similar limitations apply to the tests published by Bland et al. (2018) who evaluated 10 bicycle helmets under oblique impact conditions by using a National Operating Committee on Standards for Athletic Equipment (NOCSAE) headform coupled to a Hybrid III 50th-percentile neck. The head-neck assembly was dropped on a 30 degree inclined anvil and results have been analyzed in 
terms of linear and rotational accelerations. For the above-mentioned studies, bicycle helmet were evaluated based on global head kinematic parameters.

Under oblique impact leading to a 6D linear and angular accelerations time history, a possible way to integrate the complexity of brain geometry and brain material properties is to progress toward tissue level's brain injury criteria by using finite element models. A number of state of the art FE head models exist (Atsumi et al., 2018 ; Ganpule et al., 2017 ; Horgan \& Gilchrist, 2003 ; Ji et al., 2014 ; Kang et al., 1997; Kimpara et al., 2006; Mao et al., 2013; Sahoo et al., 2013) and some of them have been used for the definition of head injury criteria to specific injury mechanisms (Deck \& Willinger, 2008; King et al., 2003; Kleiven, 2007; Sahoo et al., 2016; Takhounts et al., 2007). These models became more powerful injury prediction tools compared to the well known global kinematic parameters like maximum rotational/linear accelerations/velocities.

The objective of the present study is to propose a new bicycle helmet test method based on linear and oblique impact tests and considering tissue level brain injury criterion computed with a finite element head model, then to apply the method to a set of 32 commercialy available bicycle helmets. The robustness of the proposed experimental versus numerical test method is verified then the capability of existing bicycle helmets to protect the brain under more realistic impact conditions and against global and tissue level brain injury criteria is expressed.

\section{MATERIAL AND METHODS}

\subsection{Experimental helmet impact conditions}

It has been demonstrated via the simulation of victims' kinematics under real world accidents that the head impact velocity presents typically a normal (radial) and a tangential component (Bourdet et al., 2012; 2016). In other terms, impacts are oblique and not just linear as suggested in current bicycle helmet standards, which consider only linear impacts (perpendicular to impacted surface). Therefore, in this study, an experimental helmet tests methodology is used that considers linear and oblique impacts. More precisely, it is proposed to conduct linear impacts against a flat horizontal anvil at a $5.5 \mathrm{~m} / \mathrm{s}$ impact velocity against FRONTAL, OCCIPITAL, and LATERAL impact points. The oblique impacts are conducted at an initial velocity of $6.0 \mathrm{~m} / \mathrm{s}$ against a $45^{\circ}$ angles anvil to the front in order to induce rotation around the $\mathrm{Y}$ axis (YRot) and two lateral impacts, one leading to $\mathrm{X}$ rotation (XRot) and one leading to $\mathrm{Z}$ rotation (ZRot). Choices of velocities used are based on existing velocity used by actual EN1078 standard to homologate bicycle helmets, and on conclusions of normal and tangential head impact velocities before impact calculated during real bicycle accident reconstructions (Bourdet et al., 2013). For the anvil's angle used for oblique impacts, several accident investigations proved that current head impact angles are between $30^{\circ}$ and $60^{\circ}$. An impact against an anvil inclined at just $30^{\circ}$ would mainly compress the foam and at $60^{\circ}$ the helmet would mainly slide along the anvil. Therefore an anvil's angle at $45^{\circ}$ is chosen in the proposed methodology. An illustration of the six experimental bicycle helmet impact tests is shown in Figure 1. 

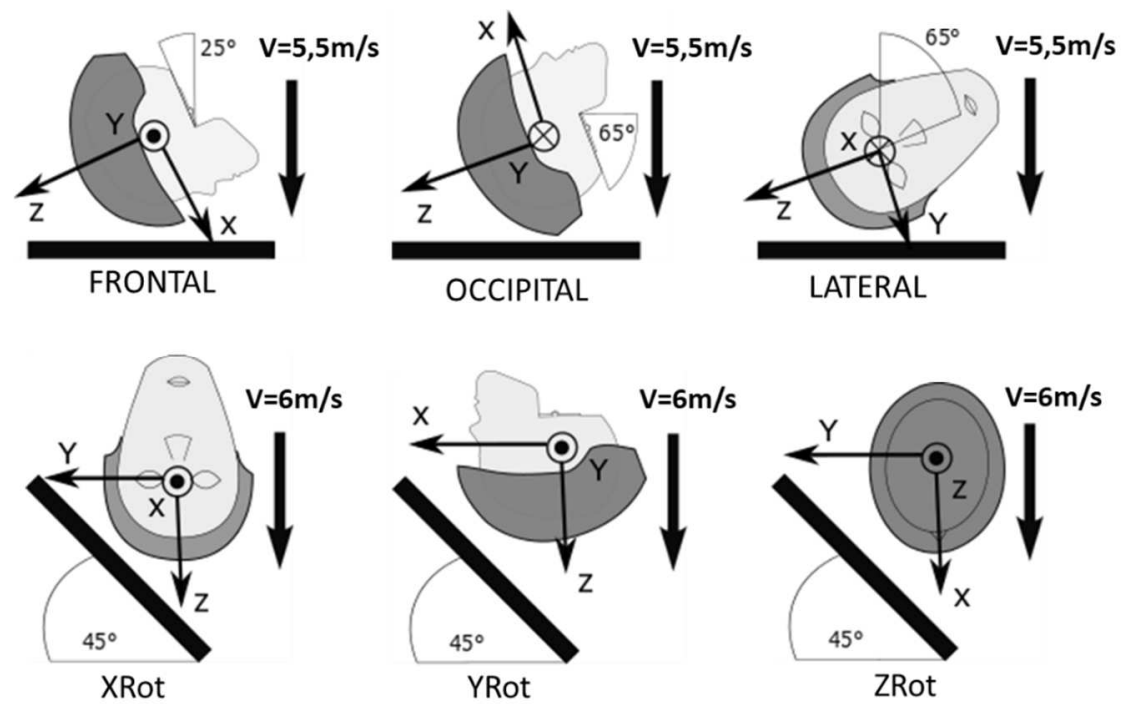

Figure 1. Illustration of the impact configurations against a horizontal anvil and the three oblique impacts against an inclined anvil.

The impacts are conducted under free fall conditions as the neck is supposed to have a limited effect on the helmet response at the time of impact (Gilchrist \& Mills, 1996). In order to control the repeatability of the experiments, each impact configuration is reproduced three times according to the test matrix reported in Table 1. For a given helmet model, 18 experimental impact tests are conducted with six helmets, nine under linear impact and nine under oblique.

Table 1. Test matrix for the three linear and three oblique impact tests involving six different helmets per helmet model.

\begin{tabular}{|c|c|c|c|}
\hline Helmet ID & First Impact & \multicolumn{1}{c|}{ Second Impact } & Third Impact \\
\hline H1 & FRONTAL & OCCIPITAL & LATERAL \\
\hline H2 & LATERAL & FRONTAL & OCCIPITAL \\
\hline H3 & OCCIPITAL & LATERAL & FRONTAL \\
\hline H4 & YRot & XRot & ZRot \\
\hline H5 & ZRot & YRot & XRot \\
\hline H6 & XRot & ZRot & YRot \\
\hline
\end{tabular}

\subsection{Test equipment}

The experimental device used to carry out the impact tests is an AD ENGINEERING drop test and consists of an aluminium column and a concrete base. It allows to guide a free fall instrumented helmeted headform, positioned on a trolley, onto an anvil with speeds up to $9 \mathrm{~m} / \mathrm{s}$. An illustration of the experimental helmet drop tests device used is proposed in Figure 2A.

For all tests, a Hybrid III $50^{\text {th }}$ headform is used in order to record both the linear and the rotational head kinematic versus time at the time of impact. This option was motivated by the fact that the Hybrid III head has more realistic rotational inertia than the EN960 headform used in EN1078 standard (2012). The Hybrid III headform is instrumented with PCB tri-axial linear accelerometers and ATA angular velocity sensors as shown in Figure 2B. Linear sensors are PCB PIEZOTRONICSinc Accelerometers $356 \mathrm{~B} 21, \pm 500 \mathrm{~g}$ with a sensitivity of $10 \mathrm{mV} / \mathrm{g}, 10.02 \mathrm{mV} / \mathrm{g}$ and $10.05 \mathrm{mV} / \mathrm{g}$ respectively for $\mathrm{x}, \mathrm{y}$ and z-axis. The ATA angular velocity sensors are a RS-06 and 06S Triaxial MHD Angular Rate sensor Arrays with a sensitivity of $50 \mathrm{mV} / \mathrm{rad} / \mathrm{sec}$ for the three channels.

The helmet drop tests lead to results in terms of three headform linear acceleration curves and three angular velocity curves for each impact. The acquisition was carried out with a sampling frequency of $50 \mathrm{kHz}$ and the signals were filtered with a CFC1000 filter. 

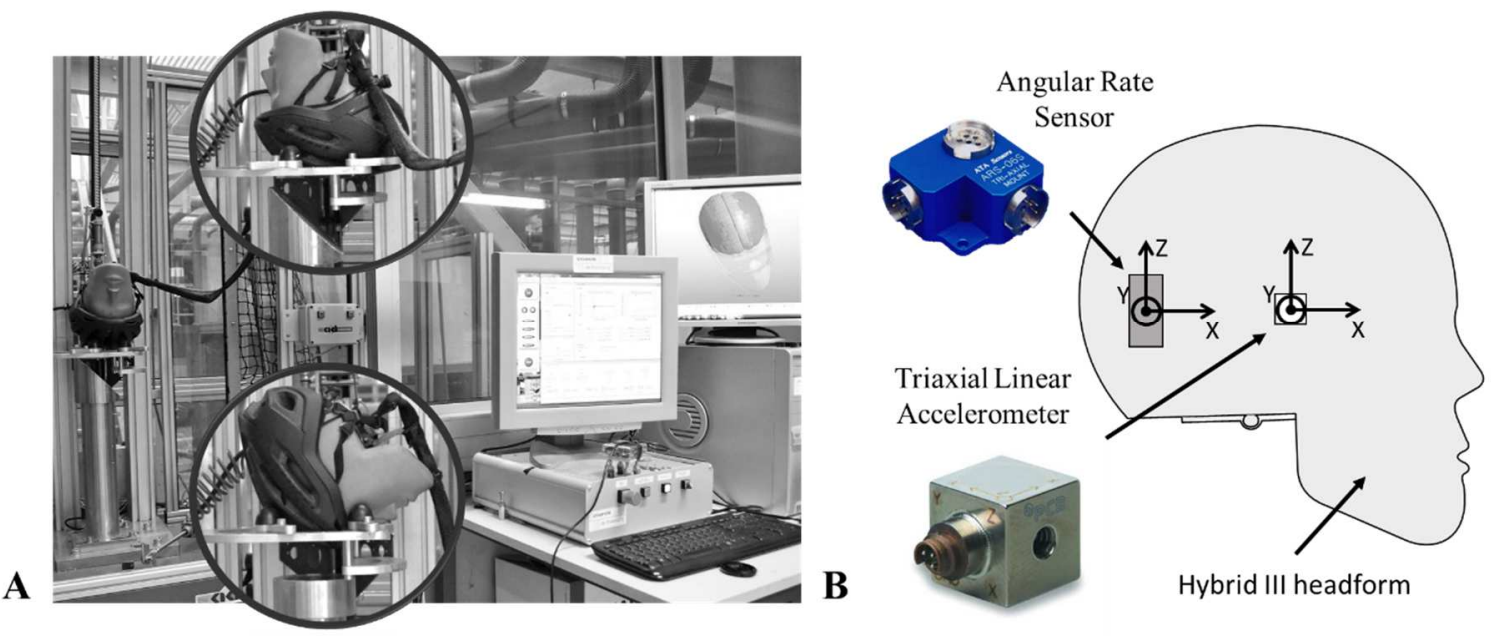

Figure 2. A. Illustration of the experimental helmet drop tests device with the two anvils used (horizontal and inclined), B. Hybrid III 5Oth dummy head equipped with linear accelerometers and angular rate sensors.

\subsection{Global and local brain injury criteria}

In order to assess the head protection performance of a helmet, both global kinematic parameters as well as local tissue level brain injury metric will be considered. The global kinematic parameters extracted from the headform response are the maximum linear acceleration, maximum angular velocity, maximum angular acceleration, HIC and BrIC values (Takhounts et al., 2013) calculated with Eq 1 and 2, respectively.

$$
H I C=\max \left[\left(\frac{1}{t_{2}-t_{1}} \int_{t_{1}}^{t_{2}} a(t) d t\right)^{2.5}\left(t_{2}-t_{1}\right)\right]
$$

With $t_{1}$ and $t_{2}[\mathrm{~ms}]$ any two points in time during any interval in the impact and a(t) the resultant linear acceleration recorded at the center of gravity of the head.

$$
B r I C=\sqrt{\left(\frac{\omega_{\mathrm{x}}}{\omega_{\mathrm{xC}}}\right)^{2}+\left(\frac{\omega_{\mathrm{y}}}{\omega_{\mathrm{yC}}}\right)^{2}+\left(\frac{\omega_{\mathrm{z}}}{\omega_{\mathrm{zC}}}\right)^{2}}
$$

Where $\omega_{\mathrm{x}}, \omega_{\mathrm{y}}$, and $\omega_{\mathrm{z}}$ are maximum angular velocities about $\mathrm{X}$-, Y- and Z-axis respectively. $\omega_{\mathrm{xC}}, \omega_{\mathrm{yC}}$, and $\omega_{\mathrm{zC}}$ are the critical angular velocities in their respective directions (Takhounts et al. (2013)).

Further the proposed helmet testing method also suggests to consider tissue level brain injury criteria derived from brain finite element modeling (FEM). Based on the 6D curves recorded during the experimental impact (three linear accelerations and three angular velocities), the helmet evaluation will be expressed in terms of head injury risk via the numerical simulation of the 18 head impacts and the relevant model based head injury criteria.

The finite element head model used in the present study is the Strasbourg University Finite Element Head Model (SUFEHM), which represents the main anatomical features included the skull, brain, brainstem, falx, tentorium, skin, and cerebrospinal fluid. More details about mechanical properties used as well as model validation have been reported in earlier studies (Deck et al., 2008; 2009; Sahoo et al., 2013; 2016). In addition to the model validation, 109 real-world head trauma cases were simulated with this head model to derive brain injury criterion in terms of intracerebral Von Mises stress to predict moderate neurological injuries or short coma. Based on an in-depth statistical analysis of different intra-cerebral parameters, it was shown that brain Von Mises stress was the most 
appropriate metric to predict short loss of consciousness also called moderate diffuse axonal injuries (mDAI). The proposed brain tolerance limit for a 50-percentage risk of mDAI has been established at $36 \mathrm{kPa}$ and the derived injury risk curve is recalled in Figure 3 (Deck \& Willinger, 2009).

The coupled experimental versus numerical bicycle helmet test method conducted in this study is illustrated in Figure 3. It considers head loadings recorded experimentally as the initial conditions of the head impact simulation with the numerical head model in order to compute the intracerebral shearing stress (Von Mises stress) and to assess the injury risk according to the injury risk curve.

For each bicycle helmet model tested, 18 experimental tests have to be performed as well as 18 numerical simulations. For each bicycle helmet model tested, seven metrics have been considered, maximum linear and rotational accelerations, maximum angular velocity, HIC and BrIC values as well as maximum brain Von Mises stress and related brain injury risks calculated.

In addition, correlations between global kinematic parameters recorded and percentage risk of moderate DAI calculated with SUFEHM have been reported.

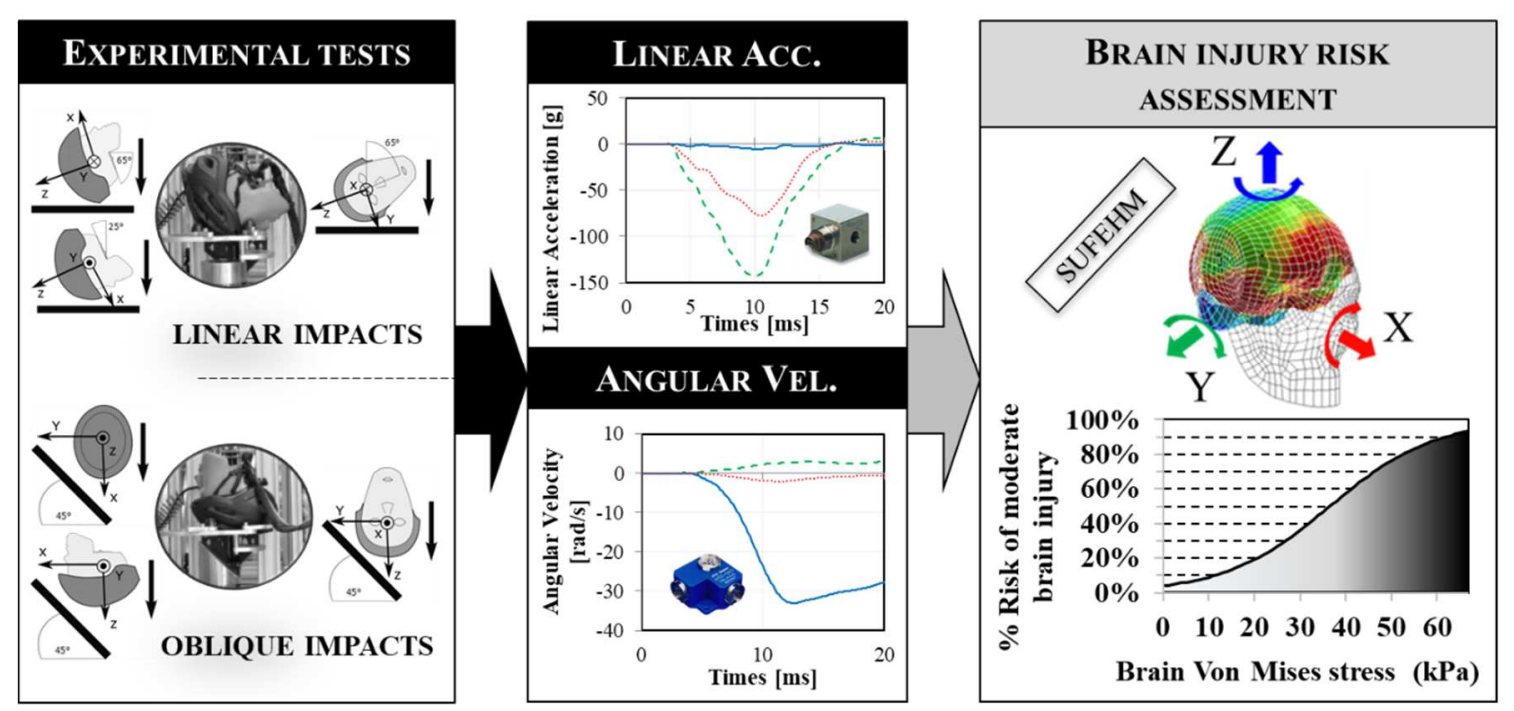

Figure 3. Illustration of the coupled experimental versus numerical bicycle helmet test method, from experimental testing to tissue level brain injury risk assessment.

\subsection{Investigation of a set of 32 bicycle helmet models}

In the present paper the exposed helmet tests method is applied to a set of 32 bicycle helmet models available on the market and listed in Table 2. For each helmet model a total of six helmets, sized M, has been purchased and submitted to the 18 impact tests mentionned in Table 1. For each single impact the headform response is considered for the extraction of global and local brain injury metrics. As each impact is reproduced three times, the total number of impacts is 576 experimental tests followed by 576 numerical simulations.

Figure 4 illustrates an example of signal values (resultant linear acceleration or angular velocity) recorded for the three same impact configurations with three different helmets for a given model. In order to evaluate the accuracy of the results, for each impact's configuration a mean standard error ( $\mathrm{SE}_{\text {mean }}$ ) will be computed by using Eq (3).

$$
S E_{\text {mean }}=\sqrt{\frac{\sum_{i=1}^{n} \sigma_{i}^{2}}{n}}
$$


Where $n$ is the number of samples, $\sigma_{i}$ the Pearson Standard Deviation of the three signals at time $\mathrm{t}_{\mathrm{i}}$ calculated with Eq (4) and $y_{i k}$ is the value of the signal for the $\mathrm{k}^{\text {th }}$ impact at time $\mathrm{t}_{\mathrm{i}}, \bar{y}_{i}$ corresponds to the mean value of the signals for the three impacts at time $t_{i}$ established with Eq (5).

$$
\begin{gathered}
\sigma_{i}{ }^{2}=\frac{1}{3} \sum_{k=1}^{3}\left(y_{i k}-\bar{y}_{l}\right)^{2} \\
\bar{y}_{i}=\frac{1}{3} \sum_{k=1}^{3} y_{i k}
\end{gathered}
$$

A relative $S R E_{\text {mean }}$ value will then be calculated by dividing $S E_{\text {mean }}$ by the maximum value of the mean signal $\left(\overline{y_{\max }}\right)$ for the linear accelerations and angular velocities signals recorded experimentally for each helmet model.

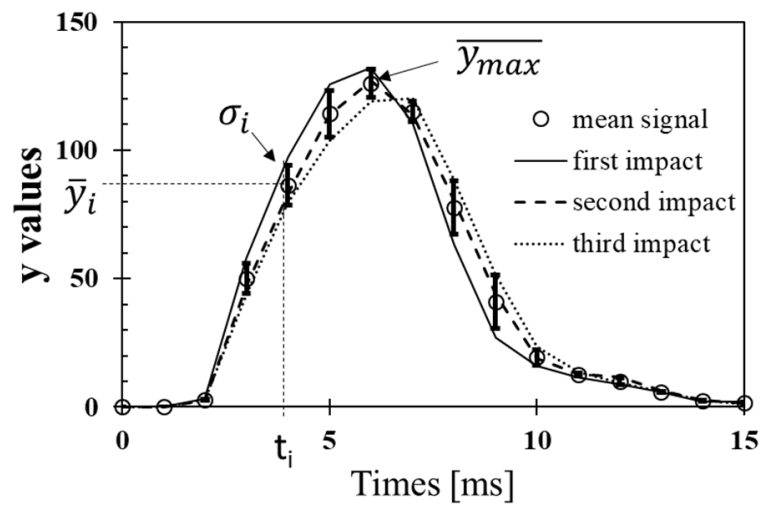

Figure 4. Representation of an example signal values (resultant linear acceleration or angular velocity) recorded for the three same impact configuration with three different helmets for a given model.

\begin{tabular}{|c|c|c|c|}
\hline Brand & Model & Size $[\mathrm{cm}]$ & Mass [g] \\
\hline \multirow{2}{*}{ ABUS } & Urban-I-2.0 & $56-61$ & 309 \\
\hline & HYBAN & $58-63$ & 460 \\
\hline ALPINA & Mythos-3.0-LE & $57-62$ & 290 \\
\hline AUTHOR & Creek-HST & $57-60$ & 380 \\
\hline$\overline{\text { BBB }}$ & BHE35-CONDOR & $58-61$ & 320 \\
\hline \multirow{2}{*}{ BELL } & STRATUS-MIPS & $58-62$ & 315 \\
\hline & ANNEX-MIPS & $58-62$ & 445 \\
\hline BONTRAGER & STARVOS-MIPS & $58-63$ & 325 \\
\hline \multirow{2}{*}{ BTWIN } & BH500 & $57-61$ & 300 \\
\hline & 100 & $58-62$ & 310 \\
\hline CANNONDALE & RADIUS & $58-62$ & $275-305$ \\
\hline CRATONI & ALLSET & $58-61$ & 290 \\
\hline CSI & FCJ-201 & $55-59$ & 541 \\
\hline GIRO & SYNTHE-MIPS & $59-63$ & 285 \\
\hline HARALD-NYBORG & BUSETTO & $58-61$ & 222 \\
\hline IKEA & SLADDA & $58-62$ & 335 \\
\hline KASK & MOJITO-16 & $59-62$ & 260 \\
\hline LAZER & REVOLUTION-MIPS & $58-61$ & 417 \\
\hline LIMAR & 555 & $57-62$ & 285 \\
\hline LIVALL & BH60 & $55-61$ & 280 \\
\hline MANGO & X-Ride & $56-62$ & $360-380$ \\
\hline MET & ESPRESSO & $54-61$ & 255 \\
\hline MUSTANG & S-282M & $52-59$ & $270-300$ \\
\hline NUTCASE & Gen3-Cherry-Blossom & $56-60$ & 454 \\
\hline OVERADE & PLIXI & $59-62$ & 490 \\
\hline
\end{tabular}

Table 2. List of the 32 bicycle's helmet models considered in this study. 


\begin{tabular}{|l|l|c|c|}
\hline \multirow{2}{*}{ SCOTT } & ARX & $59-61$ & 260 \\
\hline \multirow{2}{*}{ SCRAPPER } & SCR-S038 & $58-61$ & $250-260$ \\
\cline { 2 - 4 } & SCR-URBAN-2 & $58-61$ & $240-275$ \\
\hline SPECIALIZED & PROPERO-3 & $59-63$ & 320 \\
\hline UVEX & city-i-vo & $56-60$ & 260 \\
\hline ZERORH & ZY & $58-62$ & 290 \\
\hline
\end{tabular}

\section{RESULTS}

The resultant linear acceleration and resultant angular velocity curves obtained for the 18 impacts with one helmet model (Mustang S-282M) are reported in Figure 5. For each impact configuration, the three impacts are plotted in solid lines, dashed lines and dotted lines in order to illustrate test reproducibility. It can be observed that the discrepancy of signals for the three impacts is small whatever the impact configuration is.
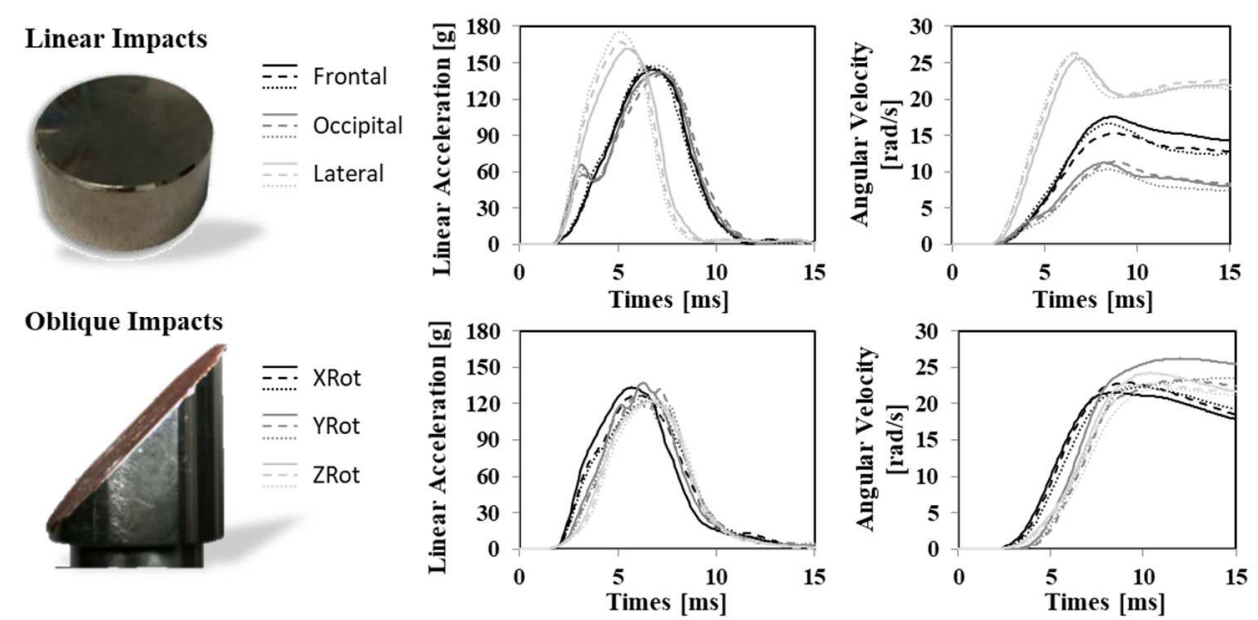

Figure 5. Resultant linear acceleration and angular velocity curves recorded for a given bicycle helmet model under three linear (upper line) and three oblique impacts (lower line) configurations. Each impact is conducted three times for reproducibility check and shown in solid, dashed and dotted lines.

Statistical results obtained in terms of $S R E_{\text {mean }}$ for the 32 helmets are reported in Figure 6 and demonstrate the robustness and reproducibility of the experimental tests for both linear and oblique impacts. $S R E_{\text {mean }}$ values calculated are less than $5 \%$ for linear accelerations and under $10 \%$ for angular velocities whatever impacts type is.
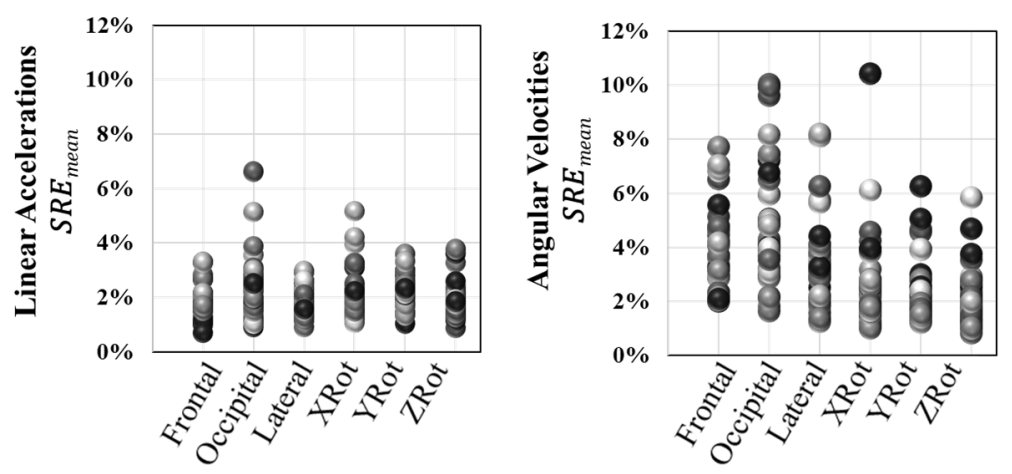

Figure 6. $S R E_{\text {mean }}$ values calculated for linear accelerations and angular velocities recorded during all impact tests and for all helmet models.

As observed on one bicycle helmet model example in Figure 5, the results typically show a clear difference in terms of angular velocity for the linear impacts, when the three oblique impacts lead to 
rotational loadings in a very similar range with a median value of about $12-25 \mathrm{rad} / \mathrm{s}$ and $25-29 \mathrm{rad} / \mathrm{s}$, respectively.

In order to assess the protection performance of a helmet, seven metrics are considered as exposed in the description of methods. Figure 7 illustrates the results in terms of box-plot view for all impact configurations and for the whole set of helmets.

As expected, the maximum linear acceleration stay below 250g for all bicycle helmets with slightly higher values for the linear impacts than for the oblique ones. Interestingly, it appears that linear accelerations are as high as $150 \mathrm{~g}$ to $190 \mathrm{~g}$ under oblique impact.

The calculated HIC values vary from 263 to 1467 with most critical values obtained for Occipital and Lateral impacts. Oblique impacts lead globally to lower values than linear ones even if a HIC value about 1,000 can be reached under YRot impact.

Coming to the angular metrics, it is essential to mention that the linear Lateral impacts lead to maximum angular velocities and accelerations, which are very similar to the oblique impacts. The maximum rotational acceleration calculated from the derivation of the angular velocity, varied from $4093 \mathrm{rad} / \mathrm{s}^{2}$ for the Occipital impacts to $8023 \mathrm{rad} / \mathrm{s}^{2}$ for Lateral impacts. Mean BrIC values varied from 0.22 for Occipital impacts to 0.63 for ZRot oblique impacts. Even if the BrIC values are higher for oblique impacts, lateral linear impacts have mean BrIC values in the same order of magnitude as for oblique impacts.

Concerning numerical simulation results involving the complex linear and angular head loadings versus time, the most critical impacts are linear-Lateral ones as well as the impact leading to rotation around the vertical axis (ZRot) with a mean Von Mises stress of around 36kPa for both configurations, which corresponds to $50 \%$ mean percentage risks of moderate DAI. Linear-Lateral impacts results conduce to a wide range of brain injury risks (from $21 \%$ to $74 \%$ ) depending on the bicycle helmet model. However, numerical results highlight that for some of the bicycle helmets low brain injury risks is observed (less than 20\%).

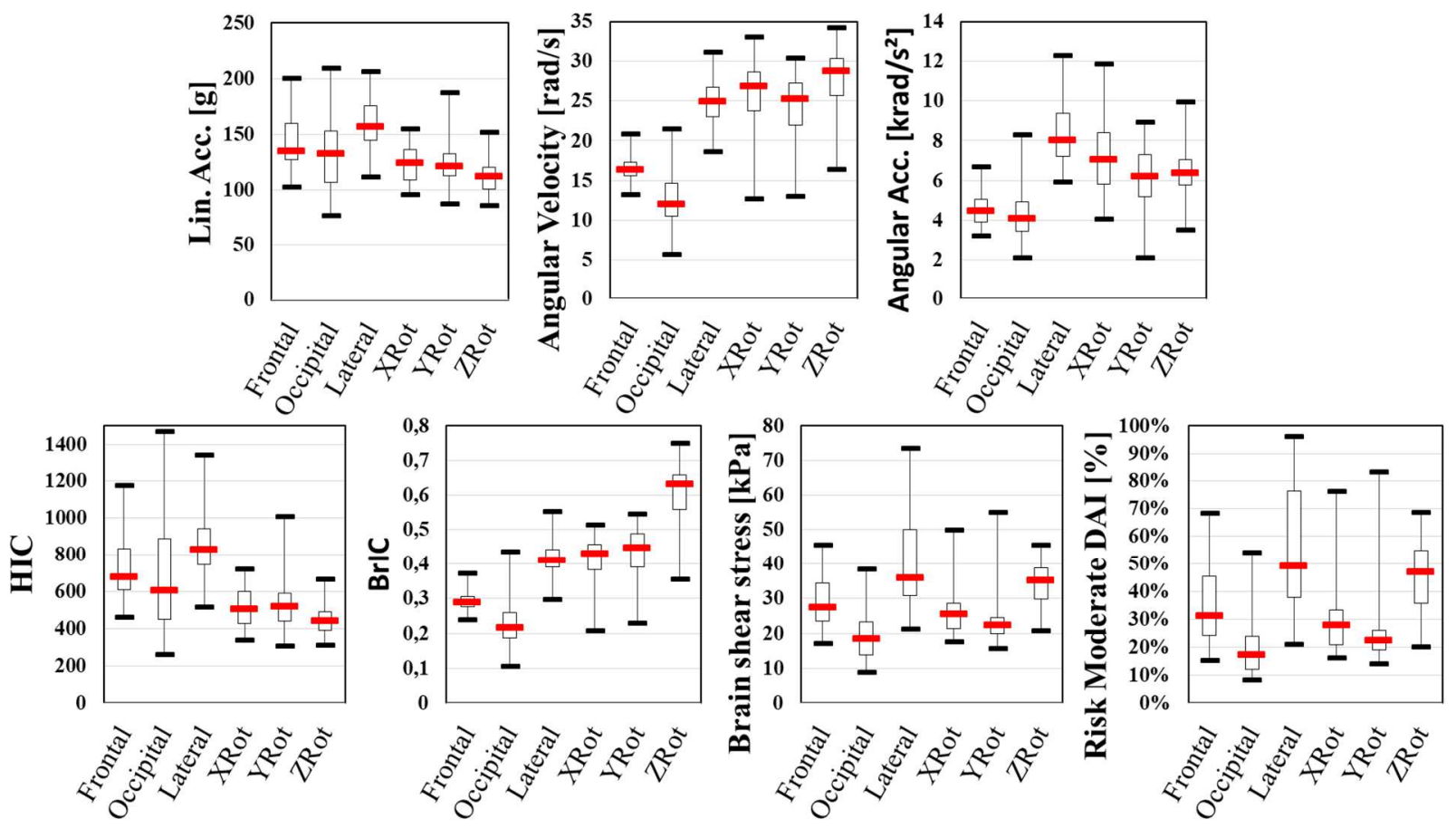

Figure 7. Representation of the results in terms of Box-plot view for Maximum linear acceleration, HIC values, Maximum angular velocity, BrIC, Maximum angular acceleration, Maximum Brain Von Mises Stress and Risk of moderate neurological injury (moderate DAI) for the full set of helmet types. 
Finally, Figure 8 shows the distribution of all impact test results in terms of global kinematic parameters (maximum linear/angular accelerations, HIC, maximum angular velocities and BrIC values) versus the percentage risk of moderate DAI calculated with SUFEHM. It appears clearly that nearly no correlation exist between any of the global kinematic parameters and the model based brain tissue level injury.

Figure 9 expresses the correlation matrix calculated for each parameters and demonstrates the low correlation between global kinematic parameters and brain Von Mises stress as well as percentage risk of moderate DAI with Spearman's coefficients less than 0.7 .

When considering only the linear metric (maximum linear acceleration and HIC value), Figure 8 shows that the most critical impact conditions are the Occipital and Lateral ones, when the tissue level injury risk is most critical for the Lateral and ZRot impacts. The angular headform response (maximum angular acceleration/velocity) shows critical values mainly for the XRot and Lateral impacts where the brain injury risk computed with the model points the ZRot and Lateral impacts. Finally, when considering BrIC value, it is the ZRot impacts that show highest values, when the computed tissue level risk is critical for the Lateral impacts.
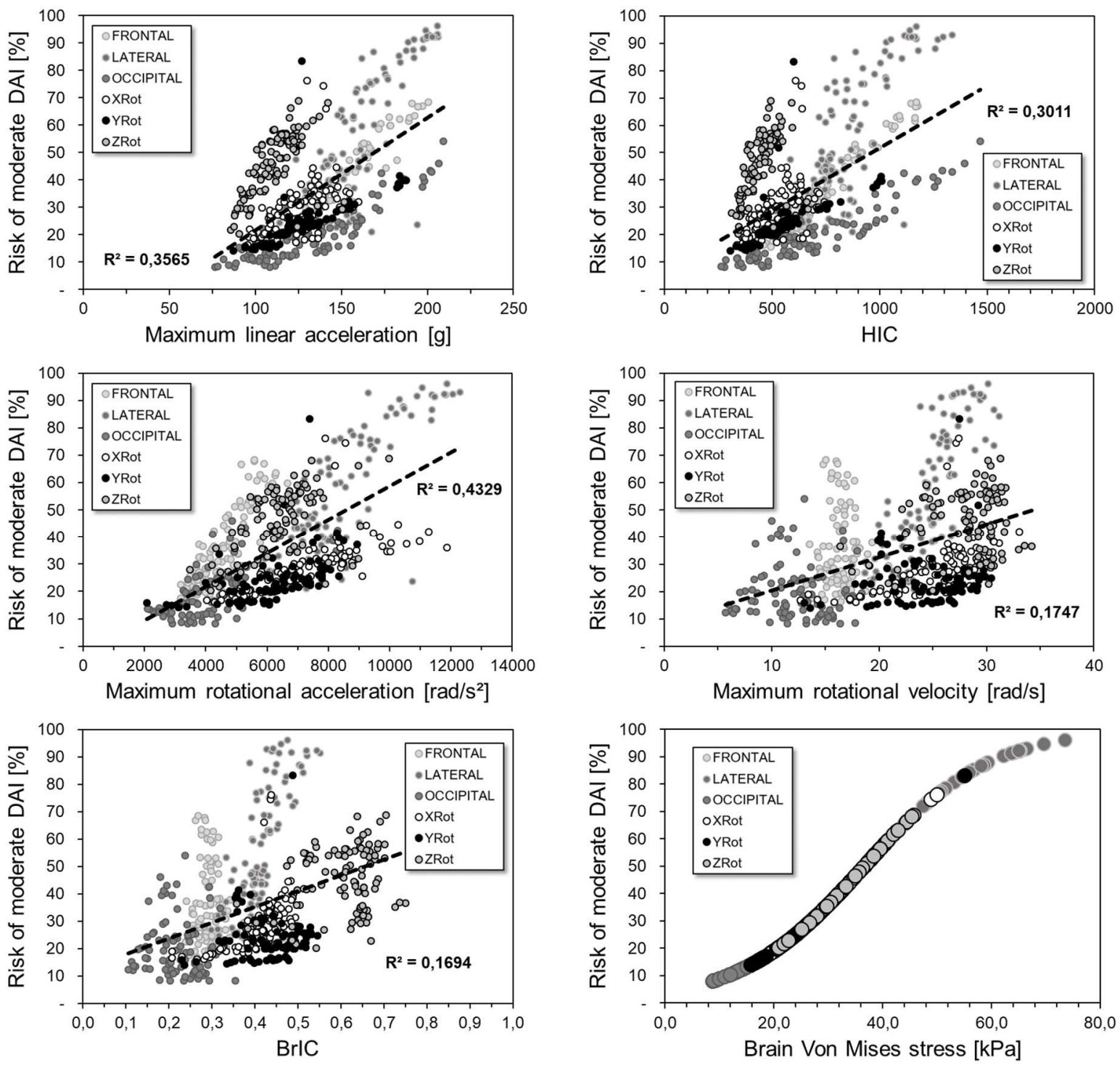

Figure 8. Distribution of all impact test results in terms of global kinematic parameters as well as brain

Von Mises stress maximum values versus percentage risk of moderate DAI calculated with SUFEHM and the corresponding Pearson's coefficient $\mathrm{R}^{2}$ calculated. 


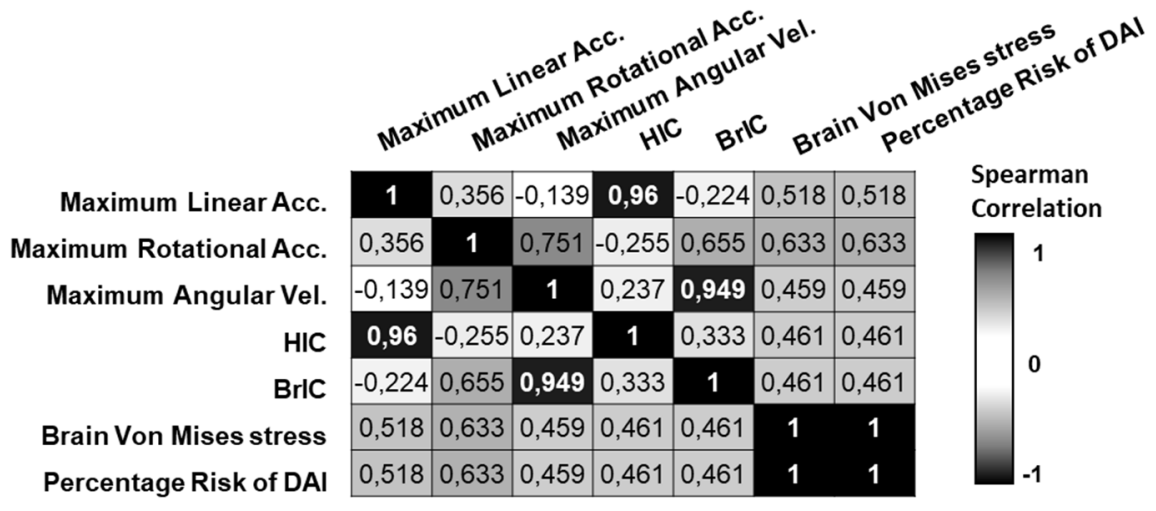

Figure 9. Correlation matrix with corresponding Spearman's coefficient calculated (a value superior to 0.7 induces a relevant correlation).

\section{DISCUSSION}

This study deals with the protection performance of a set of 32 bicycle helmets available on the market, (i.e., fulfilling the EN7810 requirements in terms of linear accelerations under linear impacts). The key objective is to evaluate helmets under more realistic impact conditions, including also the oblique impacts and against tissue level injury criterion based on finite element head modeling capable to predict moderate neurological injuries in addition to global head kinematic parameters. In the literature, global kinematic parameters are often used to assess head injury risks, especially by introducing rotational metrics (Gabler et al., 2018; Greenwald et al., 2008 ; Kimpara et al., 2011; Newman et al., 1986; 2000; Rowson et al., 2013; Takhounts et al., 2011; 2013). However, only a few of them consider injury criteria for moderate brain injury. Among them, the five most frequently considered metrics were considered in the present study and it was demonstrated that no correlation exists between any of the global kinematic parameters and the model based brain tissue level injury. The reason for this observation may be that both, linear, and angular kinematics are acting for all impacts, leading to a complex time evolution of the headform response. This phenomenon is combined with the complex brain geometry and material properties, so that finally local tissue level brain injury metric differs strongly from global parameters. As it appears that the helmet performance strongly depends on the considered metric, it is suggested for any helmet performance comparison to focus on the tissue level injury criteria as this metric takes into account the whole set of parameters involved in the complex phenomenon under study.

Bicycle helmet standards, per nature, do not differentiate performance among helmets so it is difficult for the consumer to choose a helmet based on its protection characteristics. Few first attempts of bicycle helmet rating have been published by Stigson et al. (2017), who evaluated 17 bicycle helmets under oblique impact tests. In addition, simulations were conducted using a finite element head model, and brain stains have been calculated. More recently, Bland et al. (2018) investigated differences in protective capabilities offered by 10 bicycle helmet models under so-called common real-world accident conditions. Oblique impacts were conducted onto 30 degrees from the horizontal angled anvil at $5.5 \mathrm{~m} / \mathrm{s}$ and $6.6 \mathrm{~m} / \mathrm{s}$ impact velocities. Linear and rotational accelerations were recorded and related to the helmet design. Finally, a rating system called "STAR rating" was proposed to compare helmets tested in terms of risk of concussion calculated with global kinematic parameters.

For the above-mentioned studies, no ranking based on tissue level brain injury risks has been provided in order to take into account the complexity of brain and brain loading.

Based on the results of the present study, it is possible that the development of a comparative helmet evaluation on brain injury risk can be calculated for each helmet's model. To do so, an average 
intracerebral shearing value computed for all impact configurations is calculated as expressed in Eq (6). This quantitative metric expresses the protection level of a given helmet and permits a helmet ranking from zero to five stars in steps of $1 / 2$ star, according to the head injury risk curve shown in Figure 3. The score of five stars is obtained for a mean brain injury risk under $10 \%$ and the score of $1 / 2$ star corresponds to helmet leading to a mean brain injury risk over $90 \%$. Between these two scores a scale is proposed per decrease of brain injury risks of a half star every $10 \%$ decrease of brain injury risk.

$$
\overline{\left(\sigma_{V M}\right)}=\frac{\left(\sigma_{V M_{F R O N T A L}}+\sigma_{V M_{O C C I P I T A L}}+\sigma_{V M_{L A T E R A L}}+\sigma_{V M_{X R O t}}+\sigma_{V M_{Y R o t}}+\sigma_{V M_{Z R o t}}\right)}{6}
$$

With $\overline{\left(\sigma_{V M}\right)}$ the mean maximum Von Mises Stress calculated, $\sigma_{V M_{i}}$ is the Maximum Von Mises Stress for the configuration $i$ (FRONTAL, OCCIPITAL, LATERAL, XRot, YRot and ZRot).

With this helmet-rating system, the comparative quality of the tested bicycle helmets tested is displayed in Figure 10. The proposed rating leads to a bicycle helmet's ranking from two to four stars. The proposed rating system gives a new insight to the costumer and supports manufacturers in their efforts to improve the protection of the head, under linear and oblique impacts, against tissue level brain injury.

This study tries to contribute to the objective evaluation of helmet protection capability. It suffers, however, a number of limitations and simplifications hypothesis that should be kept in mind. Concerning the impact conditions, it must be mentioned that all tests were conducted at $20^{\circ} \mathrm{C}$ and that none of the impact considered curbstone shaped anvil. Further bicycle helmets should offer adequate protection at any location when the present study only considers front, lateral, and rear impacts. Even if it has been shown that front and lateral are most frequently impacted (Bourdet et al., 2013; 2012; Ching et al., 1997; McIntosh et al., 1998; Williams, 1991), it would be of interest in future analysis to involve more impact points, hot and cold conditioning, as well as curbstone or spherical anvils.

Coming to the headform boundary conditions, the present test method suggests free headform drop tests, thus supposes that the neck has no significant effect at the time of impact. The presence of the neck obviously has an effect on the headform kinematic, especially in the second phase of the impact, where it restricts the motion of the head as it can be observed via video analysis. However, at the time of impact, when impact duration is in the 5 to $15 \mathrm{~ms}$, the neck would not affect the head response significant as the human neck presents a neutral zone of about $10-20^{\circ}$ for which rigidity is zero. In case of impact, this "initial" motion takes a few millisecond that is the order of magnitude of impact duration. This hypothesis is also confirmed by the human neck frequency analysis (Willinger et al., 2005), which showed that the neck natural frequencies are under $10 \mathrm{~Hz}$, demonstrating that there is a decoupling of the head-neck system for impacts of $10 \mathrm{~ms}$ inducing shop spectrum with energy up to $100 \mathrm{~Hz}$.

Ghajari et al $(2011$; 2013) conducted a parametric study using human body models methods and concluded that an option would be to add mass to the headform as a function of impact directions and impact angle. However, the authors also pointed the risk to lead to too stiff helmets. More recently, Feist et al. (2016) as well as Fahlstedt et al. (2016) published a parametric study using THUMS FE model and the KTH head-neck FE model respectively and showed that the neck effect on brain response lies under $10 \%$ to $15 \%$.

Few authors took the option to fit the headform to the Hybrid III dummy neck involving a hybrid head-neck system. If the effect of the Human neck on 6D head acceleration at the time of impact is still open to discussion, it is well known that the Hybrid III neck is far too rigid in flexion and not at all designed for extension, lateral flexion or torsion. A further critical aspect of the Hybrid III neck is that, due to the attachment of the lower neck extremity, no realistic rebound phenomenon is possible leading to very long impact duration (Hansen et al., 2013). 
Limitations of this study also exist at the brain injury risk assessment level. Human brain modelling is a challenging activity with its own simplification hypothesis is terms of geometry and material modelling, as well as in terms of validation only conducted in vitro. In a future step, brain injury criteria need harmonization efforts both toward model based and global injury criteria.

Finally, when it comes to helmet rating, the present proposal is based on equivalent risk of impact conditions, meaning that there is no weighting according to the higher impact occurrence of one location compared to another.

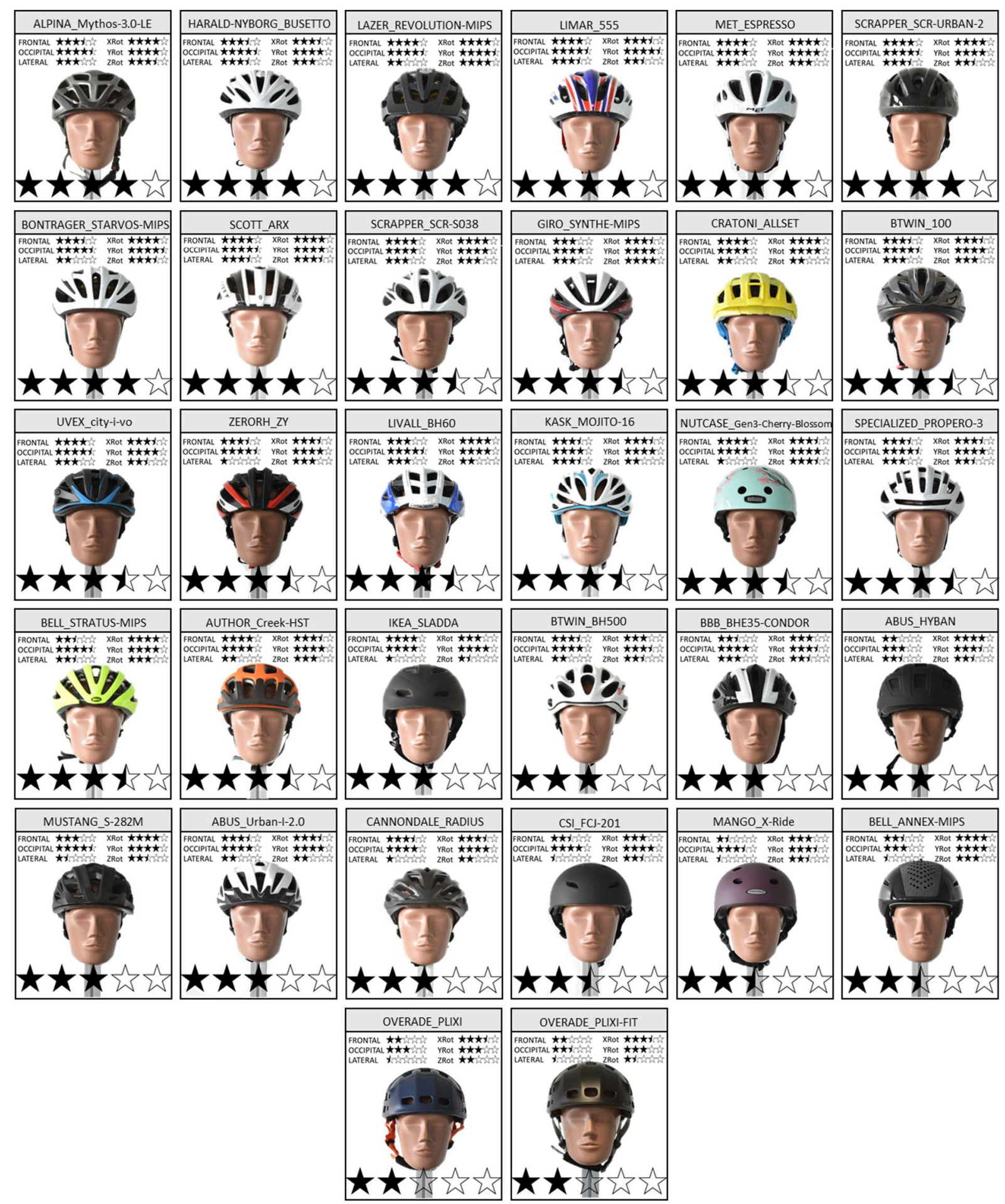

Figure 10. Display of the helmet rating in terms of stars calculated according to the brain injury risk as well as the score obtained per impacts for each bicycle's helmet model tested. 


\section{CONCLUSION}

This study presents an advanced helmet test method that considers oblique impact in addition to linear drop tests as well as brain tolerance limits based on recent biomechanical research. A coupled experimental versus numerical approach that uses the 6D headform acceleration for the numerical simulation of the brain response in order to assess the brain injury risk is applied to a set of 32 bicycle helmet models in addition to the headform's response in terms of global kinematic. Robustness of proposed experimental tests is demonstrated for all impact configurations. It is shown that the evaluation of helmet performance strongly depends on the head response metric and that none of the global kinematic parameters correlates with the injury risk computed with the head model. It is believed that the model based injury criteria is the only one that takes into account the head geometry, the brain constitutive law and the combined effect of the 6D time dependent head loading. For this reason, the injury risk computed with the head FEM is considered for the comparative evaluation of helmet performance. The derived helmet rating shows important discrepancy of the protection capability of bicycle helmets, an important information towards customers, helmet manufacturer, and standard bodies.

\section{ACKNOWLEDGEMENTS}

The authors would like to thank "Fondation MAIF" for their support.

\section{REFERENCES}

Atsumi, N., Nakahira, Y., Tanaka, E., Iwamoto M., Human brain modeling with its anatomical structure and realistic material properties for brain injury prediction, Ann Biomed Eng, Vol 46, Issue 5, pp 736-748, 2018.

Bland, M.L., McNally, C., \& Rowson, S., Differences in Impact Performance of Bicycle Helmets During Oblique Impacts, J Biomech Eng , 140(9), 091005-091005-10, 2018.

Bourdet N, Deck C, Carreira RP, Willinger R., Head impact conditions in the case of cyclist falls, Proceedings of the Institution of Mechanical Engineers, Part P: Journal of Sports Engineering and Technology, 226 (3- 4): 282-289, 2012.

Bourdet N., Deck C., Serre T., Perrin C., Llari M., Willinger R., In depth real world bicycle accident reconstructions, International Journal of Crashworthiness, 2013.

Ching RP, Thompson DC, Thompson RS, Thomas DJ, Chilcott WC, Rivara FP., Damage to bicycle helmets involved with crashes, Accid Anal Prev., 29:555-562, 1997.

Deck C., Baumgartner D., Willinger R., Influence of rotational acceleration on intracranial mechanical parameters under accidental circumstances, proceedings of the International IRCOBI Conference on the Biomechanics of Impacts, Maastricht, the Netherlands, 185-197, 2007

Deck C., Willinger R., Improved head injury criteria based on head FE model, Int. J. Crashworthiness 13 (6), 667-678, 2008.

Deck C. and Willinger R., Head injury prediction tool for predictive systems optimization. In: 7th European LS-DYNA conference, 2009.

EN1078, European Standard EN1078:2012. Helmets for Pedal and for Users of Skateboards and Roller Skates, 2012.

Fahlstedt M., Halldin P., Kleiven S., Importance of the Bicycle Helmet Design and Material for the Outcome in Bicycle Accidents, International Cycling Safety Conference, Gothenburg, Sweden, pp. 114, November 18-19, 2014. 
Fahlstedt M., Halldin P., Alvarez S.V., Kleiven S., Influence of the Body and Neck on Head Kinematics and Brain Injury Risk in Bicycle Accident Situations, proceedings of the International IRCOBI Conference on the Biomechanics of Impacts, p. 459-478, 2016.

Feist F., Klug C., A numerical study on the influence of the upper body and neck on head kinematics in tangential bicycle helmet impact, proceedings of the International IRCOBI Conference on the Biomechanics of Impacts, p. 162-184, 2016.

Ganpule S., Daphalapurkar N.P., Ramesh K.T., Knutsen A.K., Pham D.L., Bayly P.V., Prince J.L., A Three-Dimensional Computational Human Head Model That Captures Live Human Brain Dynamics, J Neurotrauma, Vol 34, Issue 13, pp 2154-2166, 2017.

Ghajari M., Galvanetto U., Iannucci L., Willinger R., Influence of the body on the response of the helmeted head during impact, International journal of crashworthiness, Vol: 16, Pages: 285-295, ISSN: 1358-8265, 2011.

Ghajari M., Peldschus S., Galvanetto U., Iannucci L., Effects of the presence of the body in helmet oblique impacts, Accident analysis and prevention, Vol: 50, Pages: 263-271, ISSN: 0001-4575, 2013.

Gennarelli T.A., Thibault L.E., Ommaya A.K., Pathophysiologic responses to rotational and translational accelerations of the head, Proceedings of the 16th Stapp Car Crash Conference, Detroit, Michigan, US, pp. 296-308, 1972.

Gennarelli T.A., Thibault L.E., Adams J.H., Graham D.I., Thompson C.J., Marcincin R.P., Diffuse axonal injury and traumatic coma in the primate, Annals of Neurology, Vol 12(6), pp 564-574, 1982.

Gilchrist A and Mill NJ, Protection of the side of the head, Accid. Anal. Prev, 28: 525-535, 1996.

Gurdjian E.S., Lissner H.R., Latimer R., Haddad B.F., Webster J.E., Quantitative determination of acceleration and intracranial pressure in experimental head injury, Neurology, Vol 3, Issue 6, pp. 417 23, 1953.

Gurdjian E.S., Lissner H.R., Photoelastic confirmation on the presence of shear strains at the craniospinal junction in closed head injury, Journal of Neurosurgery, Vol 18, pp. 58-60, 1961.

Hansen K., Dau N., Feist F., Deck C., Willinger R., Madey S.M., Bottlang M., Angular Impact Mitigation system for bicycle helmets to reduce head acceleration and risk of traumatic brain injury, Accident Analysis and Prevention, Vol 59, pp 109-117, 2013.

Holbourn A.H.S, Mechanics of head injuries, Lancet, vol. 2, pp. 438-441, 1943.

Horgan T.J. and Gilchrist M.D., The creation of three-dimensional finite element models for simulating head impact biomechanics, International Journal of Crashworthiness, vol. 8, no. 4, pp. 353366, 2003.

Ji S., Zhao W., Ford J.C., Beckwith J.G., Bolander R.P., Greenwald R.M., Flashman L.A., Paulsen K.D., McAllister T.W., Group-wise evaluation and comparison of white matter fiber strain and maximum principal strain in sports related concussion, J Neurotrauma, Vol 32, Issue 7, pp 441-54, 2014.

Kang H.S., Willinger R., Diaw B., Chinn B., Validation of a 3D anatomic human head model and replication of head impact in motorcycle accident by finite element modeling, Proceedings of the 41st STAPP Car Crash Conference, pp 329-338, 1997.

Kimpara H., Nakahira Y., Iwamoto M., Miki K., Ichihara K., Kawano S., Taguchi T., Investigation of anteroposterior head-neck responses during severe frontal impacts using a brain-spinal cord complex FE model, Stapp Car Crash J., Vol 50, pp 509-44, 2006. 
King A., Yang K., Zhang L., Hardy W., Is head injury caused by linear orangular acceleration? proceedings of the International IRCOBI Conference on the Biomechanics of Impacts, Lisbon, Portugal, 2003.

Kleiven, S., Predictors for traumatic brain injuries evaluated through accident reconstruction. In: Proceedings of the 51th Stapp Car Crash Conference, Society of Automotive Engineers, San Diego, California, Paper 2007-22-0003, 81-114, 2007.

Lissner H.R. Lebow M., Evans F.G., Experimental studies on the relation between acceleration and intracranial pressure changes in man, Surgery, Gynecology and Obstetrics, 111, p329-38, 1960.

Mao H., Zhang L., Jiang B., Genthikatti V., Jin X., Zhu F., Makwana R., Gill A., Jandir G., Singh A., Yang K.H., Development of a Finite Element Human Head Model Partially Validated With Thirty Five Experimental Cases, Journal of Biomechanical Engineering, vol. 135, no. 11, Article ID 111002, 15 pages, 2013.

McIntosh A.S., Dowdell B., Svensson N., Pedal cycle helmet effectiveness: a field study of pedal cycle accidents, Accid. Anal. Prev., 30:161-168, 1998.

McIntosh A.S., Lai A., Schilter E., Bicycle helmets: head impact dynamics in helmeted and unhelmeted oblique impact tests, Traffic Injury Prevention, Vol 14, Issue 5, pp 501-508, 2013.

McNally D.S. and Whitehead S., A computational simulation study of the influence of helmet wearing on head injury risk in adult cyclists, Accid. Anal. Prev., vol. 60, pp. 15-23, Nov. 2013.

Mills NJ, Gilchrist A., Oblique impact testing of bicycle helmets, Int J Impact Eng., Vol 35, pp 10751086, 2008.

Ommaya A.K., Goldsmith W., Thibault L., Biomechanics and neuropathology of adult and pediatric head injury, Br. J. Neurosurg. 16 (3), 220-242, 2002.

Otte D, Injury mechanism and crash kinematics of cyclists in accidents-An analysis of real accidents, $33^{\text {rd }}$ Stapp Car Crash conference, SAE paper 892425, 1989.

Sahoo D., Deck C., Willinger R., Development and validation of an advanced anisotropic visco hyperelastic human brain FE model, Journal of the Mechanical Behavior of Biomedical Materials, Vol 33, pp 24-42, 2013.

Sahoo D., Deck C., Willinger R., Brain injury tolerance limit based on computation of axonal strain, Journal of Accident Analysis \& Prevention, Vol 92, pp 53-70, 2016.

Takhounts, E. G., M. J. Craig, K. Moorhouse, J. McFadden, and V. Hasija., Development of brain injury criteria (BrIC), Stapp Car Crash J. 57:243-266, 2013.

Verschueren P., Biomechanical Analysis of Head Injuries Related to Bicycle Accidents and a New Bicycle Helmet Concept, PhD Thesis, KU Leuven, Leuven Belgium, 2009.

Weaver A.A., Danelson K.A., Stitzel J.D., Modeling brain injury response for rotational velocities of varying directions and magnitudes, Annals of Biomedical Engineering, Vol 40(9), pp 2005-2018, 2012.

Williams M., The protective performance of bicyclists' helmets in accidents, Accid. Anal. Prev., 23(23):119-131, 1991.

Willinger R., Bourdet N., Fischer R., Le Gall F., Modal analysis of the human neck in vivo as a criterion for crash test dummy evaluation. Journal of Sound and Vibration, Vol. 287, Issue 3, p. 405431, 2005. 